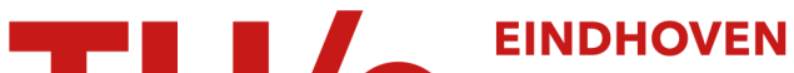 UNIVERSITY OF TECHNOLOGY
}

\section{Spatial Structure of Mn-Mn Acceptor Pairs in GaAs}

\section{Citation for published version (APA):}

Yakunin, A. M., Silov, A. Y., Koenraad, P. M., Tang, J-M., Flatté, M. E., Roy, van, W., De Boeck, J., \& Wolter, J. H. (2005). Spatial Structure of Mn-Mn Acceptor Pairs in GaAs. Physical Review Letters, 95(25), 256402-1/4. [256402]. https://doi.org/10.1103/PhysRevLett.95.256402

DOI:

10.1103/PhysRevLett.95.256402

Document status and date:

Published: 01/01/2005

\section{Document Version:}

Publisher's PDF, also known as Version of Record (includes final page, issue and volume numbers)

\section{Please check the document version of this publication:}

- A submitted manuscript is the version of the article upon submission and before peer-review. There can be important differences between the submitted version and the official published version of record. People interested in the research are advised to contact the author for the final version of the publication, or visit the $\mathrm{DOI}$ to the publisher's website.

- The final author version and the galley proof are versions of the publication after peer review.

- The final published version features the final layout of the paper including the volume, issue and page numbers.

Link to publication

\section{General rights}

Copyright and moral rights for the publications made accessible in the public portal are retained by the authors and/or other copyright owners and it is a condition of accessing publications that users recognise and abide by the legal requirements associated with these rights.

- Users may download and print one copy of any publication from the public portal for the purpose of private study or research.

- You may not further distribute the material or use it for any profit-making activity or commercial gain

- You may freely distribute the URL identifying the publication in the public portal.

If the publication is distributed under the terms of Article 25fa of the Dutch Copyright Act, indicated by the "Taverne" license above, please follow below link for the End User Agreement:

www.tue.nl/taverne

Take down policy

If you believe that this document breaches copyright please contact us at:

openaccess@tue.nl

providing details and we will investigate your claim. 


\title{
Spatial Structure of Mn-Mn Acceptor Pairs in GaAs
}

\author{
A. M. Yakunin, ${ }^{1}$ A. Yu. Silov, ${ }^{1}$ P. M. Koenraad, ${ }^{1}$ J.-M. Tang, ${ }^{2}$ M. E. Flatté, ${ }^{2}$ W. Van Roy, ${ }^{3}$ J. De Boeck, ${ }^{3}$ and J. H. Wolter ${ }^{1}$ \\ ${ }^{1}$ COBRA Inter-University Research Institute, Eindhoven University of Technology, \\ P.O. Box 513, NL-5600MB Eindhoven, The Netherlands \\ ${ }^{2}$ Optical Science and Technology Center and Department of Physics and Astronomy, University of Iowa, Iowa City, Iowa 52242, USA \\ ${ }^{3}$ IMEC, Kapeldreef 75, B-3001 Leuven, Belgium \\ (Received 23 May 2005; published 13 December 2005)
}

\begin{abstract}
The local density of states of Mn-Mn pairs in GaAs is mapped with cross-sectional scanning tunneling microscopy and compared with theoretical calculations based on envelope-function and tight-binding models. These measurements and calculations show that the crosslike shape of the Mn-acceptor wave function in GaAs persists even at very short Mn-Mn spatial separations. The resilience of the Mn-acceptor wave function to high doping levels suggests that ferromagnetism in GaMnAs is strongly influenced by impurity-band formation. The envelope-function and tight-binding models predict similarly anisotropic overlaps of the Mn wave functions for Mn-Mn pairs. This anisotropy implies differing Curie temperatures for $\mathrm{Mn} \delta$-doped layers grown on differently oriented substrates.
\end{abstract}

DOI: 10.1103/PhysRevLett.95.256402

The properties of dilute ferromagnetic semiconductors, such as $\mathrm{Ga}_{1-x} \mathrm{Mn}_{x} \mathrm{As}$, depend sensitively on the nature of the spin-polarized holes introduced into the host by the magnetic dopants $[1,2]$. Considerable controversy persists about the nature of isolated magnetic dopants in many semiconducting hosts (e.g., Mn dopants in GaN). Measurements of the local density of states (LDOS) near an individual $\mathrm{Mn}$ substituted for a $\mathrm{Ga}$ atom in $\mathrm{GaAs}\left(\mathrm{Mn}_{\mathrm{Ga}}\right)$ by cross-sectional scanning tunneling microscopy $(X$-STM) [3] have resolved this question for $\mathrm{Ga}_{1-x} \mathrm{Mn}_{x}$ As: there is a hole state bound to the Mn dopant, yielding a $\mathrm{Mn}^{2+} 3 d^{5}+$ hole complex [4-7] that produces an extended, highly anisotropic LDOS. The anisotropic shape of the bound hole state at distances $\gtrsim 1 \mathrm{~nm}$, originating from the cubic symmetry of GaAs [8] suggests highly anisotropic Mn-Mn interactions [9-11].

The ferromagnetic properties of $\mathrm{Ga}_{1-x} \mathrm{Mn}_{x}$ As, however, depend on whether this shape persists for concentrations $x$ of Mn impurities for which $\mathrm{Ga}_{1-x} \mathrm{Mn}_{x} \mathrm{As}$ is ferromagnetic $(x \gtrsim 0.01)$ [12]. Popular models of $\mathrm{Ga}_{1-x} \mathrm{Mn}_{x}$ As assume that holes residing in a bulk GaAs-like valence band, and thus evenly distributed throughout the material, mediate the ferromagnetic interaction among Mn spins [12]. However, angle resolved photoemission spectroscopy [13] observes an impurity band near $E_{F}$ and infrared absorption measurements reveal a strong resonance near the energy of the Mn-acceptor level as well as deeper in the band gap of GaAs $[14,15]$. Furthermore, recent Raman scattering experiments suggest that a $\mathrm{Mn}^{2+} 3 d^{4}$ configuration partially occurs for $x>0.02$ [16]. Recent theories suggest significant modifications in the ferromagnetic properties of $\mathrm{Ga}_{1-x} \mathrm{Mn}_{x} \mathrm{As}$ if the holes reside in a strongly disordered impurity band [11,17-20]. If the Mn density is near the metal-insulator transition, and individual $\mathrm{Mn}$ dopants states are weakly hybridizing with each other, then the inhomogeneous hole density of the impurity band measured by $X$-STM near an individual Mn dopant should
PACS numbers: 71.55.Eq, 73.20.-r, 75.30.Hx, 75.50.Pp

closely resemble that of an isolated neutral $\mathrm{Mn}$, only weakly perturbed by Mn-Mn interactions. Here we present experimental evidence that the shape of a Mn acceptor in a Mn-Mn pair remains anisotropic and retains the crosslike shape of a single Mn even when the dopants are separated by only $0.8 \mathrm{~nm}$, which is the typical distance in $\mathrm{Ga}_{0.96} \mathrm{Mn}_{0.04} \mathrm{As}$. The overlapping $\mathrm{Mn}$ wave functions at such short Mn-Mn separations are exceptionally hard to disentangle in bulk GaMnAs [21-23]. In the Mn $\delta$-doped layers of Fig. 1, however, isolated pairs and dense groups of Mn acceptors at these distances can be analyzed separately from surrounding dopants.

The measurements were performed on several samples using chemically etched tungsten tips. The $\delta$-doped layers were grown at $370{ }^{\circ} \mathrm{C}$ by molecular beam epitaxy on a $100 \mathrm{~nm} \mathrm{GaAs}$ buffer on a Zn-doped (001) GaAs substrate. The high growth temperature was chosen to suppress the

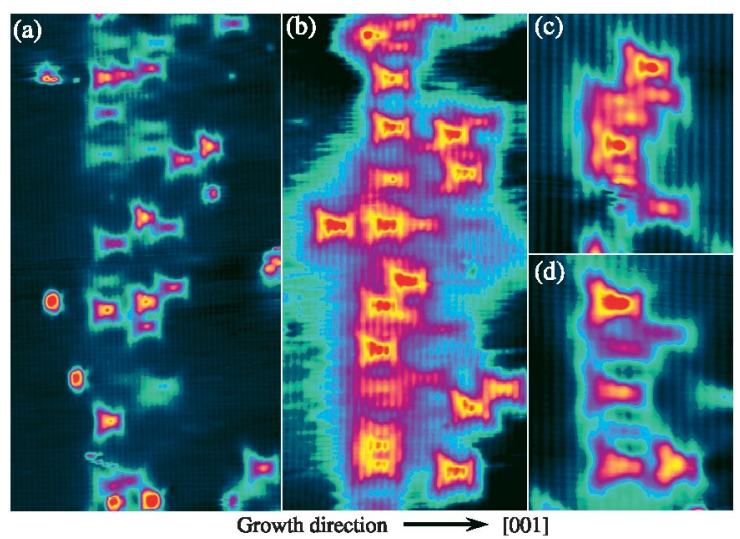

FIG. 1 (color). $X$-STM constant-current images of Mn $\delta$-doped GaAs with intentional $\mathrm{Mn}$ concentrations of $3 \times$ $10^{13} \mathrm{~cm}^{-2}$; (a) $51 \times 29 \mathrm{~nm}^{2}$; (b) $43 \times 20 \mathrm{~nm}^{2}$; (c) $12 \times 10 \mathrm{~nm}^{2}$; (d) $13 \times 10 \mathrm{~nm}^{2}$. Images were acquired at a sample bias of $U_{s}=$ $+1.5 \mathrm{~V}$ on a cleaved (110) surface. Note appearance of continuous background DOS around the layer in (b). 
appearance of structural defects such as As antisites, which would complicate Mn identification. The higher growth temperature also led to increased segregation, which broadened the $\delta$-doped layers of Fig. 1. Despite the high growth temperature a low density $\left(<10^{17} \mathrm{~cm}^{-3}\right)$ of As antisites was observed and clearly identified as charged $n$-type donors (not shown in the figure). The $\delta$-doped layers themselves clearly showed $p$-type conductivity in tunneling $I(V)$ spectroscopy. The topographies were measured with a room temperature UHV-STM $(P<2 \times$ $10^{-11}$ torr) on an in situ cleaved (110) surface.

Figure 2(a) shows the electronic topography of one of the pairs in the ionized state (the other observed pairs are similar). In the ionized configuration, it is impossible to distinguish locations of the two dopants. The potential from the double charge of the two ionized dopants induces an apparent round elevation 1.7 times larger than that of a single ionized $\mathrm{Mn}$ under the same imaging conditions $[8,24]$. In the neutral configuration (positive sample bias), however, the presence of two dopants can be clearly identified. We also found that appearance of $\mathrm{Mn}-\mathrm{Mn}$ pair wave functions is independent of sample bias down to the flatband conditions. Two examples of close, clearly identifiable Mn-Mn pairs illustrate the resilience of the Mn wave
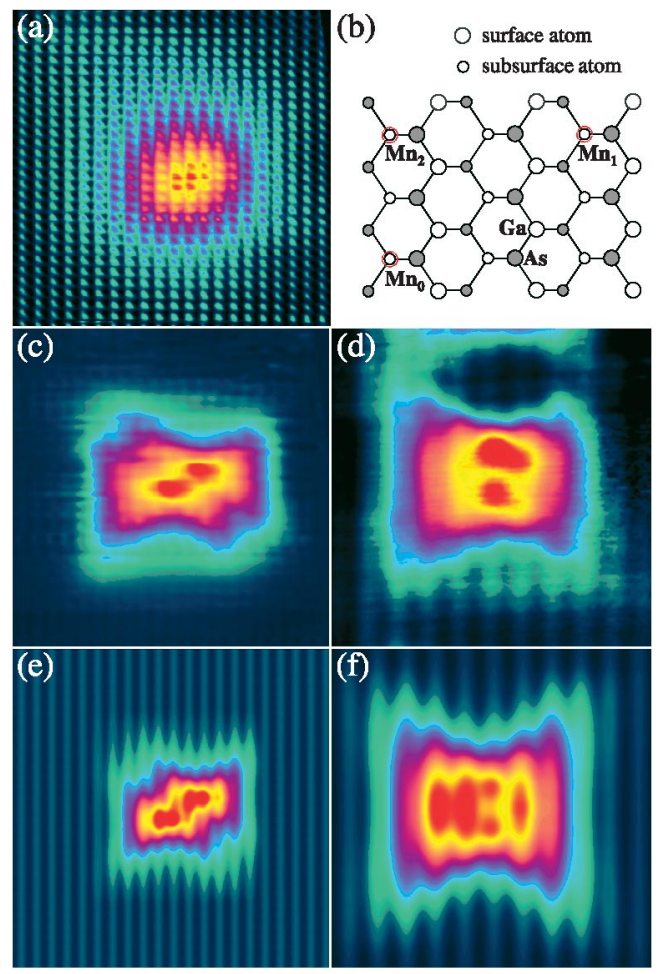

0

FIG. 2 (color). Topography of Mn-Mn pairs: (a) $12 \times 12 \mathrm{~nm}^{2}$, $X$-STM image of two ionized Mn separated by $1.4 \mathrm{~nm}\left(U_{s}=\right.$ $-0.6 \mathrm{~V})$; (b) schematic of the (110) surface showing the location of subsurface Mn; (c) same area as (a), imaged with $U_{s}=$ $+1.1 \mathrm{~V}$ so the $\mathrm{Mn}$ are neutral; (d) $6 \times 6 \mathrm{~nm}^{2}, X$-STM image of two $\mathrm{Mn}$ separated by $0.8 \mathrm{~nm}\left(U_{s}=+1.55 \mathrm{~V}\right)$; (e), (f) calculation with TBM of (c), (d), with parallel spins. function to interaction with nearby Mn dopants [Fig. 2(c) and 2(d)].

A schematic model of the GaAs (110) surface is shown in Fig. 2(b). The lateral positions of the two Mn of the first pair [shown in Fig. 2(a), 2(c), and 2(e) and separated by $1.4 \mathrm{~nm}]$ are indicated by $\mathrm{Mn}_{0}$ and $\mathrm{Mn}_{1}$; the Mn dopants themselves are located in the same subsurface layer (most likely in the fifth layer), and are well separated from neighboring Mn dopants. The topographic heights of isolated $\mathrm{Mn}$ dopants diminish in a discrete fashion as the $\mathrm{Mn}$ dopant is located in deeper subsurface layers [25]. The subsurface positions of the two Mn atoms in the pair can be determined from their individual topographic heights. The other pair [shown in Fig. 2(d) and 2(f) and separated by $0.8 \mathrm{~nm}$ ] is indicated in Fig. 2(b) by $\mathrm{Mn}_{0}$ and $\mathrm{Mn}_{2}$, and is likewise in the fifth subsurface layer. This combination has the smallest separation of those Mn-Mn pairs that we were able to identify. These measurements show that in the neutral state, the wave functions of the two Mn acceptors retain their crosslike shape even when they are separated by a distance smaller than the wave function's effective Bohr radius $a_{0} \approx 0.9 \mathrm{~nm}$.

Figure 2(e) and 2(f) shows the topography calculated with the tight-binding model (TBM) $[8,9]$ for the two pairs shown in Fig. 2(c) and 2(d) and for Mn spins parallel to each other. The calculation is averaged over the orientation of the two parallel Mn spins relative to the crystal's lattice. Qualitatively the crosslike shape is clearly evident, and the agreement between the calculations and the measurements is as good as found for a single Mn dopant in Ref. [8]. The TBM is based on the deep level model of Vogl and Baranowski [26] and is applied to a bulklike Mn acceptor. The dangling $s p^{3}$ bonds from the nearest-neighbor As hybridize with the $\mathrm{Mn} d$ states of $\Gamma_{15}$ character. The antibonding combination of these becomes the Mnacceptor state. Coupling to the $d$ states of $\Gamma_{12}$ character is weak, and hence neglected. The hybridization strength is fully determined by the acceptor level energy.

Comparison with theoretical calculations based on the TBM permits a quantitative evaluation of the effect of the Mn-Mn interaction on the measured topography. The topography for a "noninteracting baseline" is constructed by adding together the topography of two single, isolated Mn displaced by the pair separation. This baseline is then subtracted from calculations of Mn pairs, such as in Fig. 2(e) and 2(f) with differing spin orientations. Difference plots obtained in this way are shown in Fig. 3 for (110) planes displaced by 0 and 5 monatomic layers (ML) from the $\mathrm{Mn}$ pair location. The quantity $\Delta$ is the mean square root deviation of the interacting pairs density of states (DOS) from that of the noninteracting baseline integrated over the volume, and is noted in Fig. 3 for each pair (for parallel and antiparallel spins). Even for the closest pair and parallel alignment $\Delta$ is less than $1 / 3$. In previous work the spectral and spatial differences between dopant spin pairs with parallel and antiparallel spins were predicted $[9,27]$. Here the spectra could not be measured with sufficient resolu- 


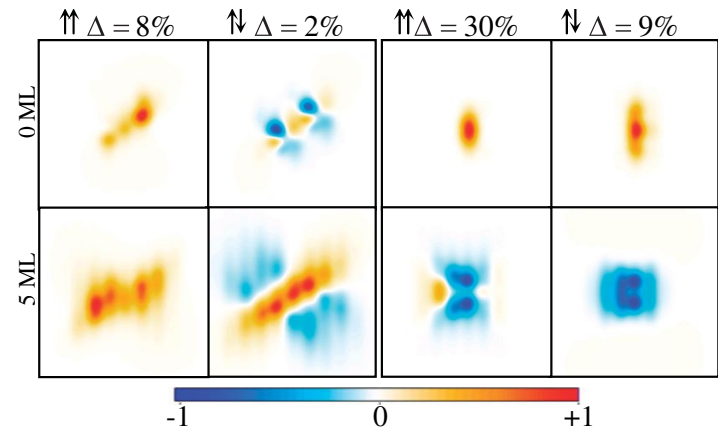

FIG. 3 (color). Calculated absolute differences between interacting and superimposed Mn-acceptor states $\left|\psi_{\text {int }}\right|^{2}-\left|\psi_{\text {sup }}\right|^{2}$ : (left set) difference for $\mathrm{Mn}_{0}-\mathrm{Mn}_{1}$ pair; (right set) difference for $\mathrm{Mn}_{0}-\mathrm{Mn}_{2}$ pair. Relative spin orientations of $\mathrm{Mn}$ atoms are indicated by the arrows. Each plot is $6 \times 6 \mathrm{~nm}^{2}$ and normalized to the maximum difference in the plot. $\Delta$ is the root mean square difference between the pair topography and a noninteracting baseline, as defined in the text.

tion to distinguish the pair spin orientations. Expected differences between pairs with parallel and antiparallel spin orientations are of the order of $10 \%$ in the fifth subsurface layer, which is not resolvable in our measurements due to the logarithmic dependence of the STM tip displacement on the local density of states.

Now that the robustness of the anisotropic crosslike shape of the Mn hole wave function has been clearly established, we explore the implications for spin-spin coupling mediated by these hole wave functions in an impurity band. The experimental data acquired with STM are confined to a two-dimensional slice along a (110) plane of the entire three-dimensional wave function. As a result, any estimation of the directional dependence of the wave function overlap taken directly from the STM experiment would be incorrect. Instead we quantify the directionally dependent overlap of the wave functions by calculating the bulklike Mn-acceptor wave function within a four-band Luttinger-Kohn envelope-function model (EFM) as well as the tight-binding model. The EFM uses the zero-range potential model [28], including a cubic correction as suggested in Ref. [8]. The ground state of the Mn acceptor can be approximated as fourfold degenerate with a total momentum of the valence hole $F=3 / 2$ and has the symmetry of the top of the valence band $\Gamma_{8}$ [29]. We neglect possible effects caused by the presence of the (110) surface and quantum spin effects from the exchange interaction between the $\mathrm{Mn}^{2+} 3 d^{5}$ core and the hole.

The calculated radial dependence of the overlap of noninteracting Mn wave functions for three crystallographic directions is presented in Fig. 4. The graph shows a nearly exponential decay of the overlap integral with separation, but is characterized by a directionally dependent decay constant. Thus the anisotropy of the overlaps increases at larger separations between the Mn dopants. The calculated directional dependence of the overlaps of noninteracting $\mathrm{Mn}$ wave functions for various $\mathrm{Mn}-\mathrm{Mn}$ separations is

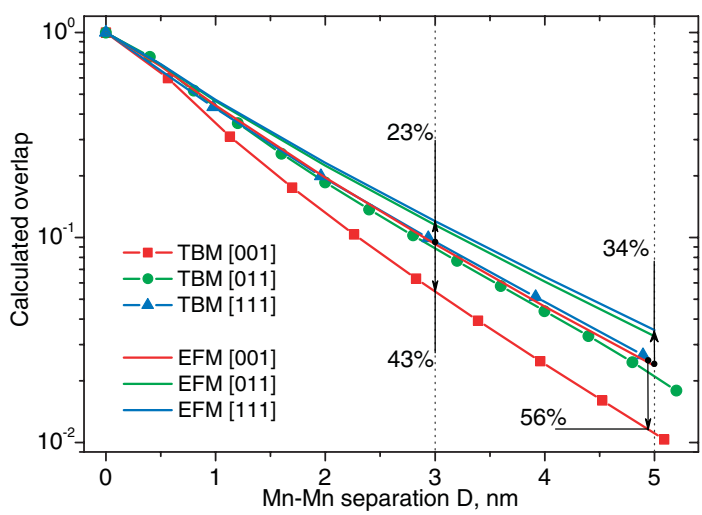

FIG. 4 (color). Separation-dependent overlap of noninteracting Mn acceptors calculated for separations along three crystallographic directions using the envelope-function model (EFM) and the tight-binding model (TBM).

shown in Fig. 5. The maximum of the overlap occurs when the impurities are located along the [111] direction, whereas the minimum occurs along the [001] direction. The TBM and EFM show similar qualitative behavior; however, the results differ slightly in value. The EFM zero-range potential model underestimates the magnitude of the wave function anisotropy compared to that observed in experiment and obtained with TBM. The anisotropy for a single bound hole is shown in a three-dimensional representation in Ref. [30].

The wave function overlap $\Omega i^{(x y z)}$ for Mn-Mn pairs grown on $(x y z)$ oriented substrates (Table I) is estimated by averaging the curves $\Sigma i$ for directions perpendicular to $(x y z)$. These calculations suggest that the wave function overlap on average is different for $\mathrm{Mn} \delta$-doped layers grown on differently oriented substrates. The density where the Mott metal-insulator transition occurs in an impurity band is determined by the overlap of localized wave functions. The anisotropic overlap of the Mn wave functions will produce a directionally dependent density threshold for percolation as well. Thus the critical concentration for the metal-insulator transition will be lower for

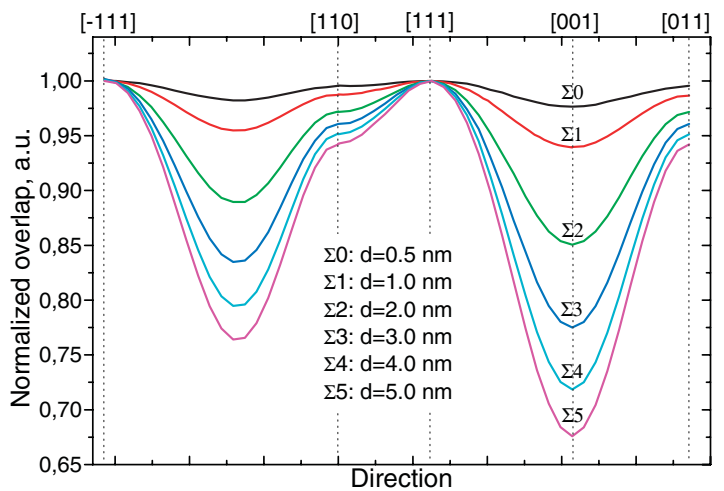

FIG. 5 (color). Directional dependence of the Mn wave function overlap for Mn-Mn separations $D$ calculated using the EFM. The curves are normalized to the maximum value $N$ given in Table I. 
TABLE I. Calculated values of the Mn wave function overlap using the EFM. $\Sigma$ is the curve number in Fig. 5, $D$ is the Mn-Mn separation, $N$ is the normalization coefficient, and $\Omega^{(x y z)}$ is the averaged overlap integral for Mn pairs grown on a (xyz) substrate.

\begin{tabular}{lccccc}
\hline \hline & $D(\mathrm{~nm})$ & $N$ & $\Omega^{(001)}$ & $\Omega^{(110)}$ & $\Omega^{(111)}$ \\
\hline$\Sigma 0$ & 0.5 & 0.654 & 0.986 & 0.990 & 0.992 \\
$\Sigma 1$ & 1 & 0.438 & 0.963 & 0.976 & 0.980 \\
$\Sigma 2$ & 2 & 0.216 & 0.912 & 0.941 & 0.952 \\
$\Sigma 3$ & 3 & 0.112 & 0.870 & 0.913 & 0.929 \\
$\Sigma 4$ & 4 & 0.060 & 0.837 & 0.892 & 0.912 \\
$\Sigma 5$ & 5 & 0.033 & 0.812 & 0.875 & 0.898 \\
\hline \hline
\end{tabular}

Mn $\delta$-doped layers grown on (111) or (110) substrates compared to $\delta$-doped layers grown on (001) substrates. As the Curie temperatures of metallic GaMnAs are much higher than those of insulating GaMnAs, the Curie temperatures and other magnetic properties for $\delta$-doped layers should be strongly dependent on the substrate orientation, with (111) substrates yielding higher Curie temperatures than (110) substrates or the currently used (001) substrates.

In conclusion, we have experimentally demonstrated that the crosslike shape of the Mn persists in groups of Mn with short Mn-Mn separation. This strongly supports the picture of impurity-band conduction and spin-spin coupling at Mn doping densities corresponding to ferromagnetic GaMnAs. We suggest that the anisotropy of the Mn wave function will substantially influence the carrier density of the Mott metal-insulator transition in $\mathrm{Mn}$ $\delta$-doped layers grown on differently oriented substrates. We expect that Mott transition will occur at lower Mn concentrations in layers grown on (111) substrates and at higher concentrations in layers grown on (001) substrates, suggesting higher Curie temperatures for (111)-grown than (001)-grown $\delta$-doped layers. These results have broad implications for all acceptor-acceptor interactions in zinc blende semiconductors, and especially for hole-mediated ferromagnetic semiconductors.

This work was supported by the Dutch Foundation for Fundamental Research on Matter (FOM), the Belgian Fund for Scientific Research Flanders (FWO), the EC GROWTH project FENIKS (G5RD-CT-2001-00535), and the ARO MURI DAAD-19-01-1-0541.

[1] Semiconductor Spintronics and Quantum Computation, edited by D. D. Awschalom, N. Samarth, and D. Loss (Springer, New York, 2002).

[2] A. H. MacDonald, P. Schiffer, and N. Samarth, Nat. Mater. 4, 195 (2005).

[3] A. M. Yakunin, A. Yu. Silov, P. M. Koenraad, W. Van Roy, J. De Boeck, and J. H. Wolter, Physica (Amsterdam) 21E, 947 (2004).

[4] T. C. Lee and W. W. Anderson, Solid State Commun. 2, 265 (1964).
[5] J. Schneider, U. Kaufmann, W. Wilkening, M. Baeumler, and F. Köhl, Phys. Rev. Lett. 59, 240 (1987).

[6] N.S. Averkiev, A.A. Gutkin, E. B. Osipov, M. A. Reshnichov, Fiz. Tverd. Tela (Ashkhabad, USSR) 30, 765 (1988) [Sov. Phys. Solid State 30, 438 (1988)].

[7] M. Linnarsson, E. Janzén, B. Monemar, M. Kleverman, and A. Thilderkvist, Phys. Rev. B 55, 6938 (1997).

[8] A. M. Yakunin, A. Yu. Silov, P. M. Koenraad, J. H. Wolter, W. Van Roy, J. De Boeck, J.-M. Tang, and M.E. Flatté, Phys. Rev. Lett. 92, 216806 (2004).

[9] J.-M. Tang and M.E. Flatté, Phys. Rev. Lett. 92, 047201 (2004).

[10] X. G. Guo, X. S. Chen, Y.L. Sun, X. H. Zhou, L.Z. Sun, J. C. Cao, and W. Lu, Phys. Rev. B 69, 085206 (2004).

[11] G. A. Fiete, G. Zaránd, and K. Damle, Phys. Rev. Lett. 91, 097202 (2003).

[12] T. Dietl, H. Ohno, F. Matsukura, J. Cibert, and D. Ferrand, Science 287, 1019 (2000).

[13] J. Okabayashi, A. Kimura, O. Rader, T. Mizokawa, A. Fujimori, T. Hayashi, and M. Tanaka, Phys. Rev. B 64, 125304 (2001).

[14] Y. Nagai, T. Kunimoto, K. Nagasaka, H. Nojri, M. Motokawa, F. Matsukura, T. Dietl, and H. Ohno, Jpn. J. Appl. Phys. 40, 6231 (2001).

[15] E. J. Singley, R. Kawakami, D. D. Awschalom, and D. N. Basov, Phys. Rev. Lett. 89, 097203 (2002).

[16] V. F. Sapega, M. Moreno, M. Ramsteiner, L. Däweritz, and K. Ploog, Phys. Rev. B 66, 075217 (2002).

[17] M. Berciu and R. N. Bhatt, Phys. Rev. Lett. 87, 107203 (2001).

[18] C. Timm, F. Schäfer, and F. von Oppen, Phys. Rev. Lett. 89, 137201 (2002).

[19] G. A. Fiete, G. Zaránd, K. Damle, and C. P. Moca, Phys. Rev. B 72, 045212 (2005).

[20] G. Bouzerar, T. Ziman, and J. Kudrnovský, Europhys. Lett. 69, 812 (2005).

[21] T. Tsuruoka, N. Tachikawa, S. Ushioda, F. Matsukura, K. Takamura, and H. Ohno, Appl. Phys. Lett. 81, 2800 (2002).

[22] J.M. Sullivan, G. I. Boishin, L. J. Whitman, A. T. Hanbicki, B. T. Jonker, and S. C. Erwin, Phys. Rev. B 68, 235324 (2003).

[23] B. Grandidier, J.P. Nys, C. Delerue, D. Stiévenard, Y. Higo, and M. Tanaka, Appl. Phys. Lett. 77, 4001 (2000).

[24] T. Tsuruoka, R. Tanimoto, N. Tachikawa, S. Ushioda, F. Matsukura, and H. Ohno, Solid State Commun. 121, 79 (2002).

[25] A. M. Yakunin, A. Yu. Silov, P. M. Koenraad, W. Van Roy, J. De Boeck, and J. H. Wolter, Superlattices Microstruct. 34, 539 (2003).

[26] P. Vogl and J. M. Baranowski, Acta Phys. Pol. A 67, 133 (1985).

[27] M.E. Flatté and D. E. Reynolds, Phys. Rev. B 61, 14810 (2000).

[28] N.S. Averkiev and S. Yu. Il'inskii, Fiz. Tverd. Tela (Ashkhabad, USSR) 36, 503 (1994) [Phys. Solid State 36, 278 (1994)].

[29] G. L. Bir and G. E. Pikus, Symmetry and Strain-Induced Effects in Semiconductors (Halsted, Jerusalem, 1974).

[30] J.-M. Tang and M.E. Flatté, Phys. Rev. B 72, 161315 (2005). 